\title{
ARTIFICATION, DÉSARTIFICATION ET RÉARTIFICATION DANS L'ARCHITECTURE CONTEMPORAINE
}

\author{
Rudy Steinmetz
}

Presses Universitaires de France | «Nouvelle revue d’esthétique »

2019/2 $\mathrm{n}^{\circ} 24 \mid$ pages 91 à 98

ISSN 1969-2269

ISBN 9782130821533

Article disponible en ligne à l'adresse :

https://www.cairn.info/revue-nouvelle-revue-d-esthetique-2019-2-page-91.htm

Distribution électronique Cairn.info pour Presses Universitaires de France.

(C) Presses Universitaires de France. Tous droits réservés pour tous pays.

La reproduction ou représentation de cet article, notamment par photocopie, n'est autorisée que dans les limites des conditions générales d'utilisation du site ou, le cas échéant, des conditions générales de la licence souscrite par votre établissement. Toute autre reproduction ou représentation, en tout ou partie, sous quelque forme et de quelque manière que ce soit, est interdite sauf accord préalable et écrit de l'éditeur, en dehors des cas prévus par la législation en vigueur en France. Il est précisé que son stockage dans une base de données est également interdit. 


\section{RUDY STEINMETL}

\section{Artification, désartification et réartification dans l'architecture contemporaine}

Le $\mathrm{XX}^{\mathrm{e}}$ siècle a connu l'émergence de nouveaux paradigmes architecturaux. Le modernisme, le postmodernisme et le déconstructivisme furent parmi les plus marquants d'entre eux. Ils ont enclenché des processus inédits d'artification, de désartification et de réartification qui ont entraîné un bouleversement de nos habitudes constructives et une refonte de nos dispositions esthétiques. Ce sont ces processus que le développement qui suit se propose d'examiner.

S'entame, avec le modernisme, un vaste procès de désartification des pratiques architecturales traditionnelles. Tout commence avec Walter Gropius. Successeur de Henry van de Velde à la tête de l'École des Arts et Métiers de Weimar, directeur de l'Académie des Beaux-Arts de cette même ville, il procède, en 1919, à la fusion de ces deux établissements. Le Bauhaus en résultera avec, pour ambition, le décloisonnement des arts, de l'artisanat et de l'industrie et la mise en œuvre «d'une architecture totale ${ }^{[1]}$ » capable d'étendre ses prérogatives aussi bien sur le moindre objet quotidien, le moindre ustensile, la moindre pièce de mobilier, que sur la ville tout entière. Cette architecture totale, prédit Gropius, donnera lieu à " une culture nouvelle ${ }^{[2]}$ » réconciliant l'art et la vie, mettant le premier au service de la seconde, et fera " naître une attitude sociale unifiée, fondée sur l'intégrité de la structure sociale, indispensable à une évolution culturelle ${ }^{[3]} »$.

Pour parvenir à réaliser l'idéal démocratique auquel aspirait le fondateur du Bauhaus, il était indispensable, à le suivre, que la mécanisation investisse le domaine de la construction. La production mécanisée devait concourir à l'éclosion d'une architecture standardisée, rationnelle, rigoureuse, aux formes
1. Walter Gropius, «Apollon dans la démocratie " (1956), in Apollon dans la démocratie/La Nouvelle Architecture et le Bauhaus, Bruxelles, Éditions françaises La Connaissance, 1969, p. 20.

2. Ibidem, p. 16 .

3. Idem. 
4. Walter Gropius, «Le Bauhaus et la nouvelle architecture » (1935), in Apollon dans la démocratie, op. cit., p. 109.

5. Ibidem, p. 108

6. Ibidem, p. 144.

7. Ibidem, p. 123

8. Ibidem, p. 106

9. Ibidem, p. 128

10. Ibidem, p. 106. simples, produite à bas coûts, offrant à tous les mêmes facilités d'accès au logement. "La standardisation n'est pas un obstacle au développement de la civilisation, mais elle en est, au contraire, une des conditions préalables immédiates ", martelait-il ${ }^{[4]}$. Pourvu, en effet, que le «nouvel ordre mondial de la machine» ne soit pas conçu comme "une fin en soi ${ }^{[5]}$ ", mais comme un moyen destiné à assurer un logis et le bien-être à tout un chacun, il sera à même de satisfaire pleinement à la "nécessité éthique de la nouvelle architecture ${ }^{[6]}$ » envisagée par Gropius.

La mise en place de ce programme édificateur égalitaire impliquait, selon son concepteur, l'abandon des formes architecturales du passé, inadaptées aux exigences de la vie moderne. Il lui fallait donc procéder à leur désartification. Ses critiques réitérées à l'adresse des écoles d'architecture vont dans ce sens. Les académies ne proposent, à en croire Gropius, qu' " un "art de salon", complètement éloigné de la vie quotidienne ${ }^{[7]}$ ", un art agonisant. Les élèves y perdent leur temps à imiter les caractéristiques formelles des styles canonisés par l'histoire. Survit ainsi une tradition architecturale plus attentive à l'apparence des constructions que soucieuse de leurs capacités réelles à répondre aux attentes de l'urbanité contemporaine. C'est pourquoi Gropius luttait avec acharnement contre la tendance, scolaire et sclérosée, à «emprunter des styles à l'antiquité et $[\ldots]$ [à] perpétuer des prototypes historiques de décoration ${ }^{[8]}$ ». En fondant le Bauhaus, il cherchait, non à imposer des formules constructives toutes faites, « un "style Bauhaus" aurait été un aveu de défaite et un retour à cette véritable stagnation et à cette inertie dévitalisante » qu'il détectait dans la reprise stérile des vieux dogmes architecturaux, mais à «exercer [...] une influence revitalisante sur le design. Nous ne basions pas notre enseignement sur des idées préconçues de la forme, mais nous recherchions l'étincelle de la vie derrière ses formes changeantes ${ }^{[9]}{ }^{»}$.

Sous cet angle de vue où les formes sont conçues, non comme devant obéir à des codes stylistiques immuables, à des canons établis une fois pour toutes, mais comme des manifestations expressives de l'énergie vitale qui les sous-tend et se libère à travers elles, il est symptomatique de constater que le premier ouvrage majeur de Gropius ait été une usine. Y avait-il, pour lui, domaine plus emblématique que celui de l'architecture industrielle à l'heure de l'industrialisation de la construction qu'il appelait de ses vœux et que toute l'époque réclamait ? Comment faire mieux admettre que dans la réalisation d'un bâtiment dont les impératifs de la production marchande impliquent rigueur et efficacité, que ces mêmes impératifs peuvent rimer avec «la satisfaction esthétique de l'âme humaine ${ }^{[10]}$ » ? Et que cet accord de l'utilité et de la beauté doit s'imposer dans la conception de toute œuvre bâtie, dès lors qu'il y va de l'aspiration vitale de la modernité à démocratiser l'accès à un habitat conforme aux besoins des usagers? 


\section{Artification, désartification et réartification dans l'architecture contemporaine}

Le complexe de bâtiments composant l'Usine Fagus à Alfeld an der Leine, en Basse-Saxe (Allemagne), fut réalisé par Walter Gropius et son collaborateur, Adolf Meyer, entre 1911 et 1925. Le corps de l'édifice principal (1911-1913) est aujourd'hui considéré comme l'une des pièces-maitresses du fonctionnalisme moderne. Plus encore, comme son "manifeste architectural ${ }^{[11]}$ ». Il traduit de façon précoce les grands principes édificateurs dont l'enseignement du Bauhaus se fera plus tard la caisse de résonance ${ }^{[12]}:$ mince ossature porteuse en béton armé (recouverte d'un parement de briques jaunes) ; charpentes métalliques noires des grandes baies vitrées annonçant le mur-rideau des gratte-ciel américains $^{[13]}$; angles des fenêtres sans montants laissant entrer la lumière avec générosité tout en conférant grâce et légèreté à la construction. Le tout obéissant à une géométrisation précise des formes faisant de cette fabrique de moules à chaussures une structure édifiée ne possédant pas d'autre qualité plastique que celle de la pureté de son volume assujetti à une stricte fonctionnalité. Ce sont ces traits caractéristiques qui ont contribué à ériger ce bâtiment au rang de modèle dont l'architecture moderne allait se réclamer, artificialisant par là même ce qui, jusque là - le bâti industriel -, restait cantonné hors du champ artistique.

Le Corbusier se déprenait lui aussi des syntagmes constructifs préexistants pour œuvrer au déploiement d'une tendance édificatrice qui soit en osmose avec les impératifs de la société moderne. À l'instar de son alter ego germanique, il n'avait pas de mots assez durs pour stigmatiser le "mensonge ${ }^{[14]}$ » des styles qu'il s'employait tout autant que lui à désartificialiser. C'est sans relâche qu'il dénonçait le fait que "l'architecture, nourrie encore un peu partout d'esprit scolastique, boudait à l'événement moderne, boudait [...] (les) techniques nouvelles [...] (et) "habillait" encore ${ }^{[15]}$ ", autrement dit, cédait à l'appel des formes stériles et de l'ornementation onéreuse et inutile.

À l'encontre d'une architecture d'apparat dominée par l'« esprit scolastique » des académies, Le Corbusier entendait mettre en avant la pureté et la simplicité des masses volumétriques telles qu'elles se donnent à voir, par exemple, dans les usines et les silos à grains du continent nord-américain. C'est à Gropius qu'il doit d'avoir eu son attention attirée sur de telles constructions. Ce dernier lui en fit parvenir des reproductions photographiques qu'il s'empressa de publier ensuite dans Vers une architecture ${ }^{[16]}$. Véritable plaidoyer en faveur d'une transvaluation de l'acte architectural, ce texte met davantage l'accent que ne le faisait Gropius sur l'importance cruciale que revêt, de l'avis de son auteur, la machinisation des différents secteurs de la société moderne. Pour Le Corbusier, il n'y avait pas à chercher ailleurs que dans les «créations de l'industrie moderne » les " véritables œuvres d'art ${ }^{[17]}$ » issues de l'esprit audacieux de la modernité. Alors que Gropius, en jetant sur les fonts baptismaux le Bauhaus, aspirait à la coordination des activités fabricatrices (artistiques, artisanales et industrielles), Le Corbusier, de son côté, réclamait leur soumission au
RUDY STEINMETZ
11. Michel Ragon, Histoire de l'architecture et de l'urbanisme modernes, t. 2, Paris, Casterman, 1986, p. 108. Les images des œuvres évoquées ici sont facilement accessibles sur le Net.

12. "Cette usine, et les bâtiments qui me furent confiés pour l'exposition du Werkbund de Cologne en 1914, accusaient nettement les caractères essentiels de mon œuvre ultérieure » (Walter Gropius, «Le Bauhaus et la nouvelle architecture", in op. cit., p. 114).

13. Voir Michel Ragon, Histoire de l'architecture et de l'urbanisme modernes, op. cit., p. 108.

14. Le Corbusier, Vers une architecture (1923), Paris, Flammarion, 1995, p. XVIII.

15. Ibidem, p. IV.

16. Voir Walter Gropius, "Le Corbusier », in Apollon dans la démocratie, op. cit., p. 154.

17. Le Corbusier, Vers une architecture, op. cit., p. 69.

nouvelle Revue d'esthétique $n^{0}$ 24/2020 
18. Ibidem, p. 80.

19. Ibidem, p. 20.

20. Ibidem, p. 108.

21. Ibidem, p. 109.

22. Voir Louis Henry Sullivan, De la tour de bureaux artistiquement considérée (1896), Montréal, B2, 2011.

23. Stanislaus von Moos signalait que «sur la plupart des photographies de ses premières maisons, il [Le Corbusier] place une automobile au premier plan - généralement la sienne, une Voisin - et l'on en vient souvent à se demander qui, de la maison ou de la voiture, est censé mieux représenter [...] l'emblème de la vie contemporaine " (Le Corbusier, une synthèse, 1979, Marseille, Éditions Parenthèses, 2013, p. 119).

24. Le Corbusier, Vers une architecture, op. cit., p. 73 .

25. Ibidem, p. 33.

26. Ibidem, p. 25.

27. Expression faisant irruption dans l'œuvre de Le Corbusier dans le courant des années 1920.

nouvelle Revue d'esthétique $n^{0}$ 2 $4 / 2020$ seul type de "construction logique et cohérente avec le monde ambiant " que parviennent à atteindre « les ouvrages qui sortent de l'atelier ou de l'usine ${ }^{[18]}$ ». Ce n'étaient dès lors pas seulement les formes primaires (cubes, cylindres, masses rectangulaires des usines et des entrepôts à céréales américains) qui passaient, à ses yeux, pour les "magnifiques PRÉMICES du temps nouveau ${ }^{[19]}$ " dont ses œuvres se voulaient la transposition artificialisante. C'était aussi la perfection machinale des automobiles, des avions et des paquebots, laquelle devait stimuler l'architecte et l'inviter à concevoir la beauté, non en tant que principe directeur de ses projets, mais en tant que conséquence d'une intention constructive qui doit rechercher avant tout le "rendement maximum» et l'« emploi minimum de moyens ${ }^{[20]}$ ", c'est-à-dire l'efficacité et la rentabilité. Autrement dit, d'après Le Corbusier, la forme d'un bâtiment ne détenait aucune légitimité à être la condition a priori de son érection. Elle n'était que l'effet d'un problème tectonique posé méthodiquement : «La masse enveloppante n'est point préconçue, elle résulte ${ }^{[21]}$. » Manière, pour lui, de revisiter le célèbre précepte de Louis Henry Sullivan : "La forme suit la fonction ${ }^{[22]}$ ", comme cela se vérifie avec la carrosserie des automobiles, le fuselage des avions ou la coque des navires dont les silhouettes sont commandées par le fonctionnement concerté de leurs organes internes, d'une part, et, de l'autre, par le respect des lois de l'aérodynamique auxquelles obéit la conception de ces engins de transport destinés à être toujours plus rapides, plus efficaces et plus ergonomiques.

Ce n'est dès lors pas un hasard si, sur certains clichés des œuvres réalisées par Le Corbusier, figure une voiture ${ }^{[23]}$. C'est ce qui arrive dans certaines prises de vue de la Villa Church (1927-1929) ou de la Villa Savoye (1928-1931). Par cette juxtaposition est mis sciemment en évidence le principe bien connu auquel il adhérait : savoir que la maison n'est pas autre chose qu' « une machine à habiter ${ }^{[24]}$ » et l'automobile une machine à se déplacer, la disposition interne des éléments constituant pareilles machines devant gouverner et non dépendre de leur configuration externe. Ce que Le Corbusier ne cessait de rappeler en affirmant que "le plan est le générateur ${ }^{[25] ", ~ q u e ~ l ' o r d o n n a n c e m e n t ~ d e s ~}$ volumes d'un édifice dépend tout entier de sa précision et que «l'architecte a pour tâche de faire vivre les surfaces qui enveloppent ces volumes, sans que celles-ci, devenues des parasites, dévorent le volume et l'absorbent à leur

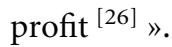

Les zones de circulation des habitations de Le Corbusier font, elles aussi, preuve de l'influence que l'automobile a pu exercer sur lui et de la promotion artificatoire qu'il lui a réservé. Éléments essentiels de l'esthétique technologique qu'il entend propager, les rampes sont très nombreuses dans ses ouvrages. Elles évoquent, bien sûr, les bretelles d'accès et de sorties des autoroutes qui l'avaient fasciné lors de son voyage aux États-Unis. Fluidifiant l'espace, elles invitent à «la promenade architecturale ${ }^{[27]}$ ": l'usager se transforme alors en "flâneur " et en spectateur de sa propre demeure ; il peut, sous divers angles de vue variant 


\section{Artification, désartification et réertification dans l'architecture contemporaine}

au gré de ses déambulations, en apprécier la logique constructive et la beauté qui s'en dégage.

De façon plus générale, les infrastructures routières sont, en tant que telles, au centre des projets et des préoccupations urbanistiques de Le Corbusier. Lorsqu'il arrive à New York en 1935, à bord du Normandie, il est confronté à un amoncellement de gratte-ciel entassés les uns sur les autres. Il a l'impression d'être devant un "spectacle alpestre ${ }^{[28]}$ " où les buildings lui apparaissent comme des "rochers hérissés ${ }^{[29]}$ ». Mais, si Manhattan lui fait l'effet d'une catastrophe, c'est néanmoins « une belle et digne catastrophe ${ }^{[30]}$ ». Il y décèle les promesses de cet urbanisme rénové auquel il aspire. Pour preuve, ce jour où, traversant le pont Georges Washington, il ne peut s'empêcher de le décrire comme un instrument presque «sacré » de précision et de perfection architectoniques dégageant sur le tissu urbain newyorkais une perspective qui en magnifie l'apparence :

De câbles et d'acier, il luit dans le ciel comme une arche retournée, bénie. [...]. Il est peint d'aluminium et l'on ne perçoit, entre ciel et eau, que cette corde fléchie appuyée sur deux pylônes d'acier aussi. Ces deux pylônes, lorsque l'auto s'élance sur la rampe, s'élèvent si haut que du bonheur vous en vient; leur structure est si pure, si ferme, si mesurée, que l'on voit enfin, ici, rire l'architecture de fer. L'auto file sur le tablier de largeur inattendue ; [...] les câbles verticaux pendent innombrables, luisant aussi dans le ciel, accrochés à cette magistrale courbe qui descend et, là-bas, remonte. Et vous voyez apparaître les tours de New York, rêve dont l'éloignement recule la brutalité ${ }^{[31]}$.

C'est aussi sur la distribution économique des espaces et des fonctions des grands navires transatlantiques reliant jadis le continent européen au continent américain que Le Corbusier s'efforce de calquer la disposition de certaines de ses villas. Autant que les voitures, c'est à une mise en valeur artificatoire des paquebots qu'il se livre dans Vers une architecture, laquelle se répercute assurément dans ses constructions. Sur les mers autant que sur terre, le déplacement est mis à l'honneur par Le Corbusier, car, pour lui, "l'architecture, c'est de la circulation $^{[32]}$ ». Ainsi, les passagers des villes flottantes vont de ponts en ponts, passent de coursives en coursives, prennent appui un moment sur les bastingages pour scruter la mer dont les flots s'étendent de tous les côtés de l'horizon, avant de reprendre leur pérégrination. De même, les propriétaires de la Villa Savoye pouvaient observer le paysage depuis le toit-terrasse de leur habitation semblable à la plate-forme supérieure d'un bateau ou depuis les longues fenêtres-bandeaux du premier étage évoquant les pans vitrés s'étirant le long des promenoirs dont les géants des mers étaient parcourus.

À partir des années 1960, le modernisme devient la cible d'une critique virulente. La contre-attaque dont il est victime émane d'architectes militant en faveur d'un retour à la tradition et d'une réintroduction du symbolisme dans l'art de construire. Robert Venturi est le premier à jeter les bases doctrinales

\section{RUDY STENMETI}

28. Le Corbusier, Quand les cathédrales étaient blanches, Paris, Plon, 1937, p. 93.

29. Ibidem, p. 78

30. Ibidem, p. 51.

31. Ibidem, p. 106.

32. Le Corbusier, Précisions sur un état présent de l'architecture et de l'urbanisme, Paris, Éditions Vincent, Fréal \& Cie, 1960, p. 48.

nouvelle Revue d'esthétique $n^{0}$ 24/2020 195 


\section{Éllobs | Atrifration/desartification}

33. Robert Venturi, De l'ambiguïté en architecture (1966), Paris, Dunod, 1999, p. 22.

34. «Pour l'artiste, créer du nouveau peut signifier choisir de l'ancien ou du déjà existant. Les artistes Pop l'ont réappris » (Robert Venturi, Denise Scott Brown, Steven Izenour, L'Enseignement de Las Vegas ou le Symbolisme de la forme architecturale, 1977, Liège, Mardaga, 1978, p. 20).

35. «Une $[\ldots]$ conséquence importante de l'art Pop concerne les méthodes de l'urbanisme. Les architectes et les urbanistes qui dénoncent avec hargne la vulgarité et la banalité du paysage urbain conventionnel proposent des méthodes élaborées destinées à abolir, ou à masquer, les éléments de pacotille des paysages actuels, ou à les exclure du lexique de leurs futures villes. Mais, pour la plupart, ils ne parviennent ni à rehausser le paysage existant, ni à lui substituer un autre parce qu'ils cherchent l'impossible » (Robert Venturi, De l'ambiguïté en architecture, op. cit., p. 52).

36. Robert Venturi, Denise Scott Brown, Steven Izenour, L'Enseignement de Las Vegas ou le Symbolisme de la forme architecturale, op. cit., p. 17

37. Ibidem, p. 27. du postmodernisme dont la visée consiste dans la réhabilitation des styles architecturaux condamnés par le modernisme. Il part d'un constat : les villes - en particulier les villes européennes qu'il connaissait bien pour avoir été boursier de l'Académie américaine à Rome de 1954 à 1956 - sont des mosaïques de façons de bâtir différentes et contrastées. La tendance moderniste récente n'est que l'une d'entre elles. Il convient dès lors de respecter ce mélange des genres qui fait la richesse des entités urbaines.

Ce que j'aime des choses, déclare Venturi, c'est qu'elles soient hybrides plutôt que "pures ", issues de compromis [...] plutôt que clairement articulées [...], accommodantes plutôt qu'exclusives [...], aussi antiques que novatrices, contradictoires et équivoques plutôt que claires et nettes ${ }^{[33]}$.

Au lieu, par conséquent, de vouloir faire table rase de la réalité citadine existante, de ce qui est " déjà là », Venturi, à l'instar des Pop artistes ${ }^{[34]}$, entend composer avec cette réalité dans ce qu'elle peut avoir de remarquable et d'ordinaire, de noble et de vulgaire ${ }^{[35]}$. Ce n'est pas l'éradication de ce qui est hétéroclite qu'il recherche au profit d'une architecture uniforme prônée par les tenants du mouvement moderne, mais sa valorisation.

Une cité américaine a procédé, quoique de manière hyperbolique, à l'artification de l'hétérogénéité urbanistique : Las Vegas.

La rue commerçante, le Las Vegas Strip en particulier [...] lance à l'architecte le défis de la regarder positivement, sans préjugés. Les architectes ont perdu l'habitude de regarder l'environnement sans jugement préconçu parce que l'architecture moderne se veut progressiste $[. .$.$] ; elle n'est pas satisfaite des conditions existantes { }^{[36]}$.

Plutôt que de fermer les yeux devant l'urbanisme kitsch de Las Vegas qui ressemble à s'y méprendre à un parc d'attraction «dysneylandesque », Venturi y décèle une source d'inspiration. Les hôtels-casinos aux décors hollywoodiens, pastiches des plus grands édifices de l'histoire universelle de l'art de bâtir, traduisent, malgré leur grandiloquence, un désir authentique de la part des êtres spatiaux que nous sommes : celui de vivre dans une "architecture [...] de communication ${ }^{[37]}$ ", en d'autres termes dans un milieu bâti qui ne les prive pas de l'iconographie historique des langages constructifs traditionnels bannis par les modernistes, mais qui, au contraire, s'emploie à les réartifier - fût-ce de façon outrancière.

Parallèlement au postmodernisme, mais en léger décalage, se développe, dans les années 1970, un autre mouvement architectural dont l'identité est malaisée à cerner : le déconstructivisme. Son acte de naissance officiel intervient tardivement, en 1988, lorsque Philip Johnson et Mark Wigley organisent au Moma, à New York, une exposition intitulée : Deconstructivist Architecture. Elle réunit huit architectes : Frank Gehry, Daniel Libeskind, Rem Koolhaas, le duo formé de Wolf 


\section{Artification, désartification et réartification dans l'architecture contemporaine}

Prix et Helmut Swieczinsky (agence Coop Himmelblau), Zaha Hadid, Peter Eisenman et Bernard Tschumi. Ils ne se reconnaissent pas tous sous cette dénomination. D'autres, n'ayant pas été conviés à l'exposition, s'en réclament. Mais, que les contours de ce mouvement - ou, devrait-on dire, de cette mouvance - soient flous, qu'il s'agisse d'une nébuleuse, il n'en reste pas moins que les projets et les travaux des architectes qui s'y rattachent ou que l'on y rattache contre leur gré relèvent d'une stratégie commune : procéder à la désartification architecturale de l'architecture dans toutes ses variantes stylistiques ${ }^{[38]}$.

Cette désartification architecturale bat en brèche aussi bien les formes pures du modernisme que les formes évocatrices du passé dont les adeptes du postmodernisme entretenaient - avec une certaine dose d'ironie pour certains - la nostalgie. Cela est notable chez Frank Gehry. En particulier dans la première de ses œuvres : l'extension de sa résidence privée (1977-1978) à Santa Monica, en Californie. Considérée comme l'une des manifestations primitives de la déconstruction dans le domaine de l'architecture, la maison personnelle de Frank Gehry est un bungalow construit en 1920 et acquis par l'architecte et sa seconde épouse en 1975. Entourée d'une annexe contenant la cuisine, la salle à manger et la terrasse, la demeure d'origine ne se laisse plus entrapercevoir qu'à travers les percées de l'enveloppe qui la recouvre. Ce qui frappe au premier coup d'œil, c'est l'absence de cohésion entre la bâtisse initiale et l'ajout plus récent qui est venu s'y greffer tel un corps étranger. C'est comme si les règles les plus élémentaires de la composition architecturale avaient été bafouées : il n'y a ni centralité ni équilibre dans la disposition des volumes, pas plus qu'il n'y a de distinction entre charges et supports, le tout donnant l'impression de former un agrégat aléatoire. Autre aspect remarquable : la "pauvreté » des matériaux employés ${ }^{[39]}$ : tôles ondulées, treillis, bois de coffrage sont autant de composants qui, en vertu de leur trivialité, font signe vers les Merzbau de Kurt Schwitters ou l'Arte povera; manière de rompre avec l'usage des matériaux traditionnels (marbre, pierre de taille, etc.) et la plasticité des matériaux neufs (fer, béton) prisés par les architectes modernes.

Ce qui justifie la dysphorie, l'apparence chaotique de cette réalisation, c'est la proximité du travail de Gehry avec celui des artistes américains des années 1960, tel que, entre autres, le néo-dadaïste Robert Rauschenberg, surtout connu pour ses Combine Paintings. Comme celui-ci, il aime s'adonner à l'accumulation d'éléments de natures différentes sans chercher à ce que l'œuvre achevée en soit la mise en ordre. Contrairement aux recommandations de Le Corbusier, il évite toute idée préconçue, tout plan rigide et reste ouvert à ce qui peut survenir de manière improbable.

Je m'essaie à des formes sur des maquettes. Je regarde, j'enlève, je recommence, et le projet évolue pas à pas. Si je réfléchis trop à l'avance, je n'ai plus de plaisir, plus de surprise, et le résultat s'en ressent. [...] Parfois, [...] je ne sais pas bien où je vais ${ }^{[40]}$.
RUDY STEINMETI

38. «La déstructuration des espaces est une manière d'exercer une critique de l'architecture par des moyens eux-mêmes architecturaux » (Michel Makarius, Ruines, 2004, Paris, Flammarion, 2011, p. 221).

39. Frank Gehry faisait déjà usage, au début des années 1970, d'un matériau banal, brut, grossier, dans la confection d'un mobilier (tables, chaises, fauteuils, etc.) réalisé à partir de cartons d'emballages pliés et collés. Son design relève, lui aussi, d'une tentative de valorisation esthétique, d'artification de ce qui reste généralement en marge de la sphère de la création.

40. Frank Gehry, "La vie comme elle vient, conversation avec Frank Gehry ", L'Architecture d'aujourd'hui, vol. 286, avril 1993, p. 83. Que le procès créateur échappe en partie à son auteur, c'est ce que Gehry indique ailleurs au sujet des transformations de sa propre maison : "You were never sure what was intentional and what wasn't. It looked in process. You weren't sure whether I meant it or not» (Frank Gehry, "Commentaries ", in Architecture + process, Gehry talks, Londres, Thames \& Hudson, 2003, p. 54).

nouvelle Revue d'esthétique $n^{0} \quad 24 / 2020$ | 97 
41. Frank Gehry, art. cit., p. 83.

42. Idem.

43. Nathalie Heinich, Roberta Shapiro (dir.), De l'artification. Enquête sur le passage à l'art, Paris, Éditions de l'EHESS, 2012, p. 278.

44. Étienne Souriau, « Passé, présent, avenir du problème de l'esthétique industrielle ", in Esthétique industrielle, Paris, Puf, 1952, p. 13.

45. Idem.

46. Ibidem., p. 7. Voir aussi la note 4 .

47. Bernard Oudin, Dictionnaire des architectes, Paris, Seghers, 1994, p. 172.

nouvelle Revue d'esthétique $n^{0}$ 24/2020
À une époque, confie-t-il dans le même entretien, je regardais les papiers froissés dans ma corbeille et j'imaginais des formes de bâtiments, ou m'attachais aux compositions accidentelles qu'ils formaient ${ }^{[41]}$.

Quant à l'aspect de chantier en cours et au sentiment de déséquilibre tectonique que procure la perception de sa maison, il se souvient :

À une certaine époque, j'ai exploré cette idée de bâtiments en construction. J'étais [...] intéressé par celle [l'idée] du mouvement figé. J'aimais l'image d'un tas de bois emporté par le vent et arrêté en pleine course ${ }^{[42]}$.

Le contexte architectural «anarchique » de Los Angeles joue aussi un rôle. Gehry, qui s'y est installé très jeune avec ses parents, a toujours voulu que ses interventions architecturales en soient le reflet et l'accentuation.

Il est légitime, pour finir, de se demander si l'on peut parler d'artification dans le domaine architectural. C'est la question que posent Nathalie Heinich et Roberta Shapiro en notant que

[la pratique constructive] a toujours été soumise à de fortes contraintes de technicité, et n'a pu se faire reconnaître comme un «art » [...] que de façon partielle. De même que les métiers d'art, l'architecture n'est pas une activité en voie d'artification mais, plutôt, en perpétuelle tension entre technique et art ${ }^{[43]}$.

Mais, pourrait-on objecter, tabler sur la persistance d'une «tension entre technique et art », n'est-ce pas donner à penser que les propriétés artistiques de l'architecture ne peuvent, voire ne doivent pas se concilier avec ses propriétés fonctionnelles? N'est-ce pas, pour formuler l'objection en termes derridiens, autoriser la possibilité d'accréditer l'idée selon laquelle il y aurait, en droit, une distinction entre le versant esthétique et le versant utilitaire de l'activité édificatrice ? Que beauté et usage sont, en celle-ci, à même d'être conçues indépendamment l'une de l'autre?

Pareille distinction - s'il y en a - n'a pas manqué de susciter des réticences. C'est le cas d'Étienne Souriau. En forgeant la notion «d'art impliqué»-pour se départir de celle «d'art appliqué ${ }^{[44]}$ »-, il cherchait à montrer que certains types de production ne supposent pas l'adjonction d'une part artistique qui leur soit extrinsèque, mais l'incluent en eux de manière immanente. Cette portion d'art qui leur est inhérente "ne peut être mise à part que par la pensée, car elle fait intimement partie de ce [genre de] travail créateur ${ }^{[45]}$ ». Une telle observation n'entre «nullement en conflit avec les normes fondamentales de l'art, nommément architectural ${ }^{[46]} »$. N'était-ce pas, du reste, sur l'incongruité qu'il y aurait à dissocier le beau de l'utile que les partisans du modernisme fondaient leur approche de l'œuvre édifiée ? Plus fondamentalement et comme le fait remarquer à juste raison Bernard Oudin, ne convient-il pas d'admettre, avec lui, que "l'adjectif "fonctionnel" ne saurait désigner la caractéristique de telle école du | $98 \mathrm{XX}^{\mathrm{e}}$ siècle, mais bien la qualité première et nécessaire de toute architecture ${ }^{[47]}$ » ? 